\title{
AVALIAÇÃO DO DESEMPENHO DO DIÂMETRO DOS CORPOS MOEDORES NO PROCESSO DE MOAGEM DE BOLAS DA AGA-CUIABÁ
}

\author{
W. L. GOMES ${ }^{1}$, T. L. A. JATOBÁ ${ }^{1}$, H. DELBONI JR. ${ }^{1}$, L. P. R. GOMES ${ }^{2}$, T. A. V. SILVA
Escola Politécnica da Universidade de São Paulo ${ }^{1}$, AngloGold Ashanti S/A $^{2}$
wellingtonlacerdagomes@gmail.com ${ }^{1}$ \\ Submetido 21/11/2017 - Aceito 22/11/2017 \\ DOI: $10.15628 /$ holos.2017.6585
}

\section{RESUMO}

O circuito industrial de moagem de bolas da AGA-Cuiabá, Sabará-MG, consiste de um circuito direto fechado com hidrociclones. Um estudo para redução do diâmetro dos corpos moedores de reposição, com o intuito de promover uma maior fragmentação no interior do moinho e, consequentemente, geração de um produto mais fino ou aumento da produção, por meio da redução da carga circulante. O presente trabalho tem como objetivo avaliar o desempenho do moinho de bolas da AGA-Cuiabá operando com diferentes diâmetros de corpos moedores. O estudo foi baseado em duas abordagens, uma industrial na qual foi estabelecido um diagnóstico atualizado e detalhado do desempenho do circuito industrial. A base para tal diagnóstico foi uma amostragem global do circuito, a partir da qual foram realizadas calibrações de modelos matemáticos de processo para cada um dos equipamentos, bem como a integração dos mesmos para representar a operação típica do circuito, tal plataforma foi denominada de Caso Base. A segunda consistiu em ensaios de moagem em batelada, com diferentes distribuições de corpos moedores. Deste modo, a partir do Caso Base foram simulados cenários com redução do diâmetro de corpos moedores de reposição do circuito industrial, os quais foram comparados com os resultados obtidos nos ensaios em laboratório a fim de consolidar e validar os resultados simulados. Portanto, o trabalho apresentou um método para avaliar o desempenho do diâmetro de corpos moedores em moinho de bolas baseado em modelagem matemática e simulação, assim como em ensaios de moagem em batelada.

PALAVRAS-CHAVE: cinética de fragmentação, moagem em batelada, simulação.

\section{ASSESSMENT OF BALL SIZE DIAMETER PERFORMANCE IN MILLING BALL PROCESS OF AGA-CUIABÁ}

\begin{abstract}
The AGA-Cuiabá ball mill industrial circuit, Sabará-MG, consists of a direct circuit closed with hydrocyclones. A study was carried out to reduce the diameter of mill balls in order to improve fragmentation inside the mill, consequently, generate finer product or increase production by reducing circulating load. The present work aims to evaluate the performance of AGA-Cuiabá ball mill operating with different diameters of balls. The study was based on two approaches, the first one was industrial in which an updated and detailed diagnosis of the performance of the industrial circuit was established. Basis for such a diagnosis was a global sampling of the circuit, from which calibrations of
\end{abstract}

mathematical process models were performed for each equipment, as well as their integration to represent the typical operation of the circuit, such platform was called Base Case. The second consisted in batch grinding tests, with different distributions of mill balls. Thus, from the Base Case, scenarios were simulated with reduction of the diameter of mill balls of the industrial circuit, which were compared with the results obtained in the laboratory tests in order to consolidate and validate the simulated results. Therefore, the work presented a method to evaluate the performance of mill ball diameter based on mathematical modeling and simulation, as well as laboratory batch grinding tests.

KEYWORDS: grinding kinetics, batch grinding, simulation 


\section{INTRODUÇÃO}

O circuito industrial de moagem de bolas da AGA-Cuiabá consiste de um circuito direto fechado com hidrociclones. Um estudo para melhoria de desempenho de processo de modo a promover a geração de um produto final mais fino para a etapas subsequentes de flotação e aumento da capacidade do circuito industrial foi realizado.

Conforme informações da equipe da AGA o circuito de britagem da respectiva operação trabalha com sobrecapacidade, possibilitando assim a geração de um produto mais fino, mediante o fechamento da abertura da peneira, ou aumento da produção. Deste modo, o presente trabalho teve como objetivo avaliar o desempenho de diferentes distribuições de corpos moedores dentro do moinho de bolas da AGA-Cuiabá em relação à fragmentação resultante. Normas para submissão de artigos

\section{REVISÃO DA LITERATUIRA E METODOLOGIA}

O desenvolvimento do trabalho compreendeu duas abordagens, quais sejam: uma industrial na qual foi estabelecido um diagnóstico atualizado e detalhado do desempenho do circuito de moagem industrial com ênfase no moinho de bolas e, a segunda consistiu na execução de ensaios de moagem em batelada, com diferentes distribuições de corpos moedores.

A abordagem industrial fundamentou-se em uma amostragem do circuito industrial, a partir da qual foram realizadas calibrações de modelos matemáticos de processo para cada um dos equipamentos, assim como a integração dos mesmos para representar a operação típica do circuito, tal plataforma foi denominada de Caso Base. A partir do Caso Base foram simulados quatro diferentes diâmetros de corpos moedores de reposição do moinho de bolas.

A calibração dos modelos matemáticos de processo para os equipamentos individuais, bem como a integração dos mesmos foram realizadas com o auxílio do software JKSimMet 6.1. Os modelos matemáticos utilizados para calibrar os equipamentos do circuito de moagem AGACuiabá foram o Perfect Mixing Model, PMM (WHITEN, 1976) para o moinho de bolas e o modelo de Nageswararao (1978 e 1995) para hidrociclones.

O Perfect Mixing Model é um modelo de balanço populacional, no qual assume-se que todo o material no interior do moinho está perfeitamente misturado. Deste modo, o moinho se comporta como um equipamento de transformação de granulometria onde o material alimentado na i-ésima fração mais o material moído das frações mais grossas até o tamanho da i-ésima fração é igual ao produto da i-ésima fração somado ao material fragmentado da i-ésima fração.

O modelo de Nageswararao, o qual foi desenvolvido diretamente a partir do modelo de Lynch e Rao (1975), baseia-se na curva de partição padrão de classificação e foi elaborado com base em dados experimentais obtidos a partir de testes em escala piloto. Nageswararao estimou expoentes empíricos para quatro equações referentes ao cálculo dos parâmetros, que descrevem o processo de classificação em hidrociclones, quais sejam: diâmetro mediano de partição, partição de água e de polpa ao underflow e vazão volumétrica da alimentação.

Os ensaios de moagem em batelada tiveram como objetivo comparar o desempenho quanto à fragmentação das diferentes cargas de corpos moedores simuladas em relação ao Caso 
Base com os dados experimentais. Para tanto, foram formadas cinco diferentes cargas de corpos moedores para representar o Caso Base e os quatro diâmetros de corpos moedores de reposição simulados, respectivamente.

Os ensaios de moagem foram realizados com a amostra da alimentação nova do circuito de moagem, tomada na campanha de amostragem. Foi utilizado um moinho com dimensões de 12 " $\mathrm{X}$ 12 ", dotado de barras longitudinais na face interna, as quais atuam como levantadores dos corpos moedores. As condições operacionais dos ensaios foram semelhantes ao moinho industrial. A massa de sólidos para cada ensaio foi calculada a partir do grau de enchimento do moinho (31\%), volume de vazios da carga de bolas (40\%), massa específica dos sólidos (3,0 g/cm3) e porcentagem de sólidos (78,6\%), assim cada ensaio foi executado com 4554 g de sólidos e $1240 \mathrm{~g}$ de água. 0 moinho operou com $76 \%$ da velocidade crítica.

Devido ao diâmetro dos corpos moedores de reposição estudados ( $\left.312^{\prime \prime}\right)$ serem muito grandes em relação ao moinho de laboratório utilizado nos ensaios de moagem (12"), equivalente a $29 \%$ do diâmetro do moinho, as cargas de corpos moedores empregadas nos ensaios foram formadas com diâmetros menores, porém mantendo-se a proporção dos mesmos. Com base no método de Bond (1958) foram formadas as cinco cargas de cargas de corpos moedores utilizadas nos ensaios de moagem, conforme apresentado na Tabela 1 na página 5.

De acordo com Gomes (2014) utilizou-se o tempo de moagem de 15 minutos para reproduzir as simulações com modificações dos diâmetros de corpos moedores de reposição do moinho AGA-Cuiabá. Para assegurar uma maior representatividade dos ensaios de moagem estes foram realizados em duplicata, sendo identificados como Ensaios A e B para cada uma das configurações de carga estudadas.

O procedimento de execução dos ensaios de moagem consistiu em dividir a carga de bolas e a massa de sólidos, em três alíquotas similares. O moinho foi então carregado em sequência alternada, com um terço de carga de bolas e um terço de massa de minério até que o moinho fosse todo carregado. Após este procedimento foi adicionada a massa de água necessária para que a polpa atinja a porcentagem de sólidos estipulada. O moinho foi então fechado e o ensaio iniciado, conforme ilustrado na Figura 1 na página seguinte.

\section{RESULTADOS E DISCUSSÃO}

O objetivo de se adotar um Caso Base consistiu em se determinar um diagnóstico da operação atual do circuito de moagem da AGA-Cuiabá, estabelecendo assim as referências que balizaram os cenários simulados. $O$ Caso Base do circuito de moagem consistiu de moinho de bolas $(5,0 \times 7,8 \mathrm{~m})$, operando com $31 \%$ de grau de enchimento e $76 \%$ da velocidade crítica. A potência aplicada ao moinho foi de $3.203 \mathrm{~kW}$. A etapa de classificação operou com 7 hidrociclones de 20", com vortex e apex de $140 \mathrm{~mm}$ e $100 \mathrm{~mm}$, respectivamente, e pressão de operação de $58 \mathrm{kPa}$. Há dois Knelson XD40 instalados, os quais processam aproximadamente $20 \%$ da carga circulante. Assim o concentrado Knelson é enviado à fundição e o rejeito retorna à caixa de bomba da alimentação da classificação. A Figura 2, aseguir, ilustra o fluxograma de processo do circuito de moagem e concentração gravimétrica da AGA-Cuiabá e o sumário do balanço de massas para o Caso Base. 


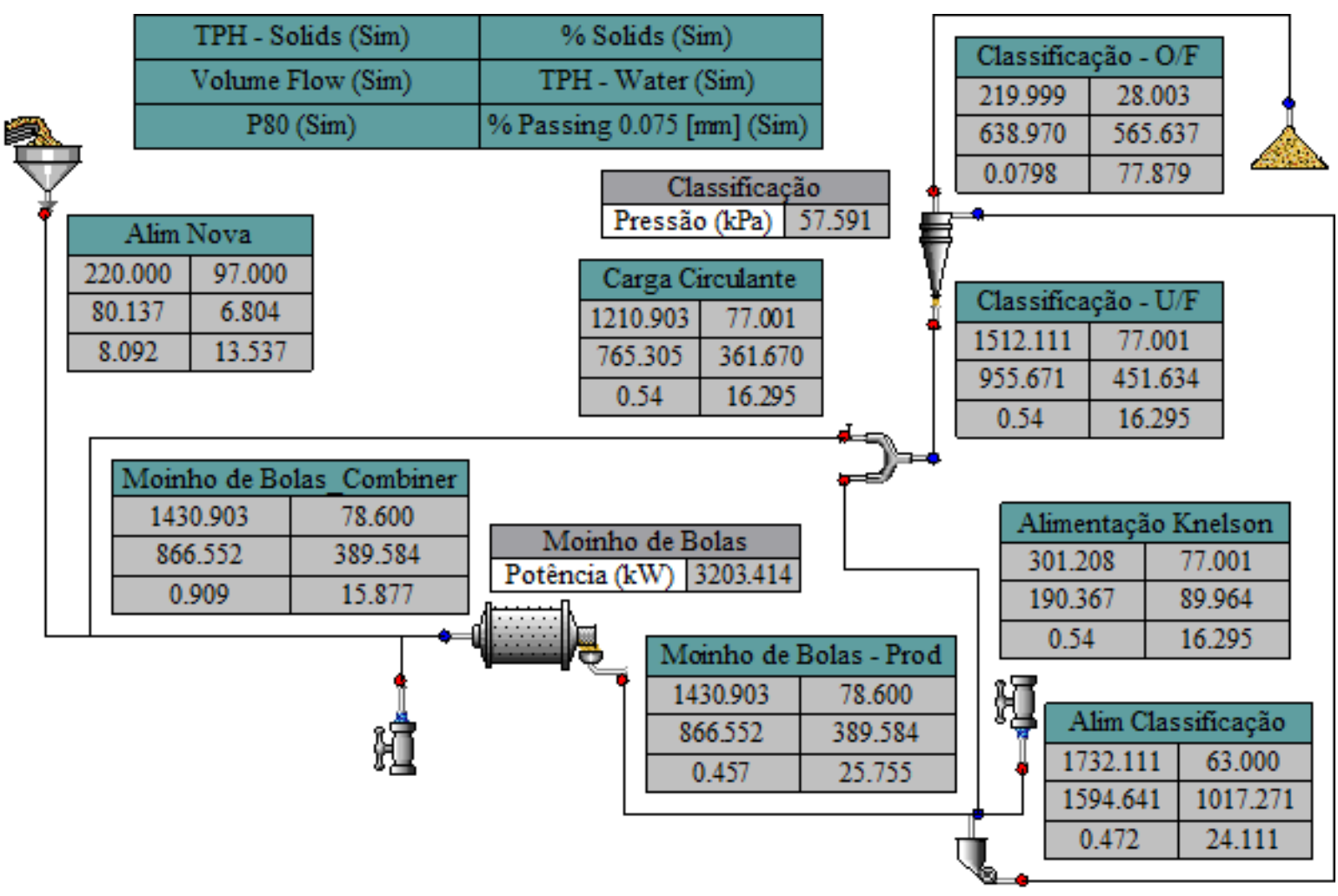

Figura 1. Fluxograma de processo do circuito industrial de moagem - Caso Base.

\subsection{Caso Base}

Os resultados do Caso Base para o circuito de moagem indicaram:

- $\quad$ O Caso Base apresentou baixa eficiência de classificação de finos para a malha 0,075 mm (41\%). A baixa eficiência da etapa acarretou em alta carga circulante (687\%), fato que dificulta a operação e limita o aumento de capacidade do circuito;

- $\quad$ O moinho de bolas indicou alta cinética de fragmentação para partículas maiores que 1,68 $\mathrm{mm}$, em contrapartida indicando redução da cinética de fragmentação para partículas menores que $1,68 \mathrm{~mm}$.

Foram destacados dois pontos com possibilidade de melhoria, quais sejam:

- Melhoria da etapa de classificação de modo a aumentar a sua eficiência, reduzindo o bypass de finos e de água e, como resultado a carga circulante;

- Redução do diâmetro de corpos moedores de reposição, de modo a promover maior fragmentação das partículas $<1,68 \mathrm{~mm}$ e a geração de um produto final mais fino. 
BRASIL \& NORTE (2017)

HOLDS

Tabela 1. Distribuição das cargas de corpos moedores dos ensaios de moagem em laboratório.

\begin{tabular}{|c|c|c|c|c|c|c|c|c|c|c|c|c|c|c|c|}
\hline \multirow{2}{*}{$\begin{array}{l}\text { Tamanho } \\
(\mathrm{mm})\end{array}$} & \multicolumn{3}{|c|}{$\begin{array}{c}\text { Distribuiçôes Originais para } \\
\text { cada Diâmetro Máximo (\%) }\end{array}$} & \multirow{2}{*}{$\begin{array}{c}\text { Tamanho } \\
\text { Original } \\
(\mathrm{mm})\end{array}$} & \multirow{2}{*}{$\begin{array}{c}\text { Tamanho } \\
\text { Empregado } \\
(\mathrm{mm})\end{array}$} & \multicolumn{2}{|c|}{$\begin{array}{c}\text { Caso Base: } \\
20 \% 31 / 2^{\prime \prime}-80 \% 3 "\end{array}$} & \multicolumn{2}{|c|}{$\begin{array}{c}\text { Simulado: } \\
10 \% 31 / 2^{\prime \prime}-90 \% 3 "\end{array}$} & \multicolumn{2}{|c|}{$\begin{array}{c}\text { Simulado: } \\
20 \% 31 / 2^{\prime \prime}-80 \% 21 / 2^{\prime \prime}\end{array}$} & \multicolumn{2}{|c|}{ Simulado: 100\% 3" } & \multicolumn{2}{|c|}{$\begin{array}{c}\text { Simulado: } \\
20 \% 3 "-80 \% 21 / 2^{\prime \prime}\end{array}$} \\
\hline & $90 \mathrm{~mm}$ & $75 \mathrm{~mm}$ & $65 \mathrm{~mm}$ & & & $\%$ Mássica & $\begin{array}{l}\text { Número } \\
\text { de Bolas }\end{array}$ & $\%$ Mássica & $\begin{array}{l}\text { Número } \\
\text { de Bolas }\end{array}$ & $\%$ Mássica & $\begin{array}{l}\text { Número } \\
\text { de Bolas }\end{array}$ & $\%$ Mássica & $\begin{array}{l}\text { Número } \\
\text { de Bolas }\end{array}$ & \% Mássica & $\begin{array}{l}\text { Número } \\
\text { de Bolas }\end{array}$ \\
\hline 90 & 24,0 & 0 & 0 & $90^{*}$ & $\Rightarrow 63,5$ & 4,8 & 2 & 2,4 & 1 & 4,8 & 2 & 0 & 0 & 0 & 0 \\
\hline 75 & 38,0 & 31,0 & 0 & $75^{\star}$ & 57,2 & 32,4 & 14 & 31,7 & 14 & 7,6 & 3 & 31,0 & 13 & 6,2 & 3 \\
\hline 65 & 20,5 & 39,0 & 34,0 & $65^{*}$ & 50,8 & 35,3 & 21 & 37,2 & 22 & 31,3 & 19 & 39,0 & 24 & 35,0 & 21 \\
\hline 50 & 11,5 & 19,0 & 43,0 & 50 & 38,1 & 17,5 & 25 & 18,3 & 26 & 36,7 & 53 & 19,0 & 28 & 38,2 & 55 \\
\hline 40 & 4,5 & 8,0 & 17,0 & 40 & 30,0 & 7,3 & 21 & 7,7 & 22 & 14,5 & 42 & 8,0 & 23 & 15,2 & 45 \\
\hline 25 & 1,5 & 3,0 & 6,0 & 25 & 15,0 & 2,7 & 63 & 2,9 & 67 & 5,1 & 119 & 3,0 & 70 & 5,4 & 126 \\
\hline Total & 100 & 100 & 100 & & Total & 100 & 146 & 100 & 152 & 100 & 238 & 100 & 158 & 100 & 250 \\
\hline
\end{tabular}
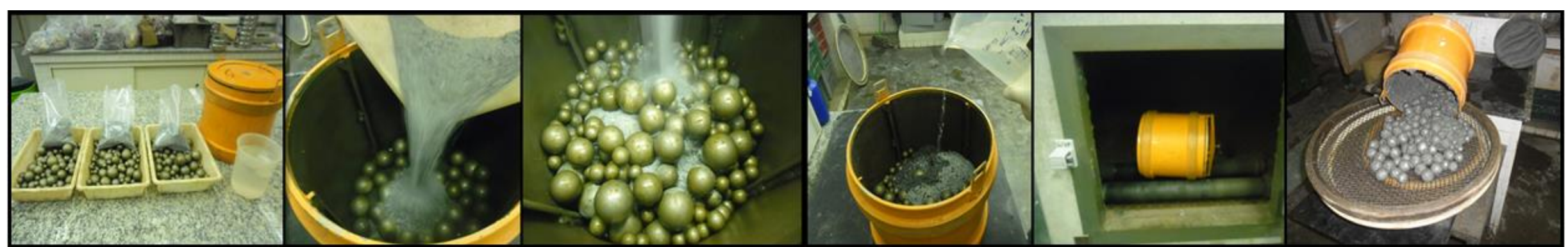

Figura 2. Sequência de operações dos ensaios de moagem. 


\subsection{Cenários Simulados}

A Figura 3, a seguir, apresenta as curvas de cinética de fragmentação para cada uma das configurações de diâmetros de corpos moedores de reposição simulados, comparando-as com as distribuições granulométricas de cada um dos fluxos conectados ao moinho.

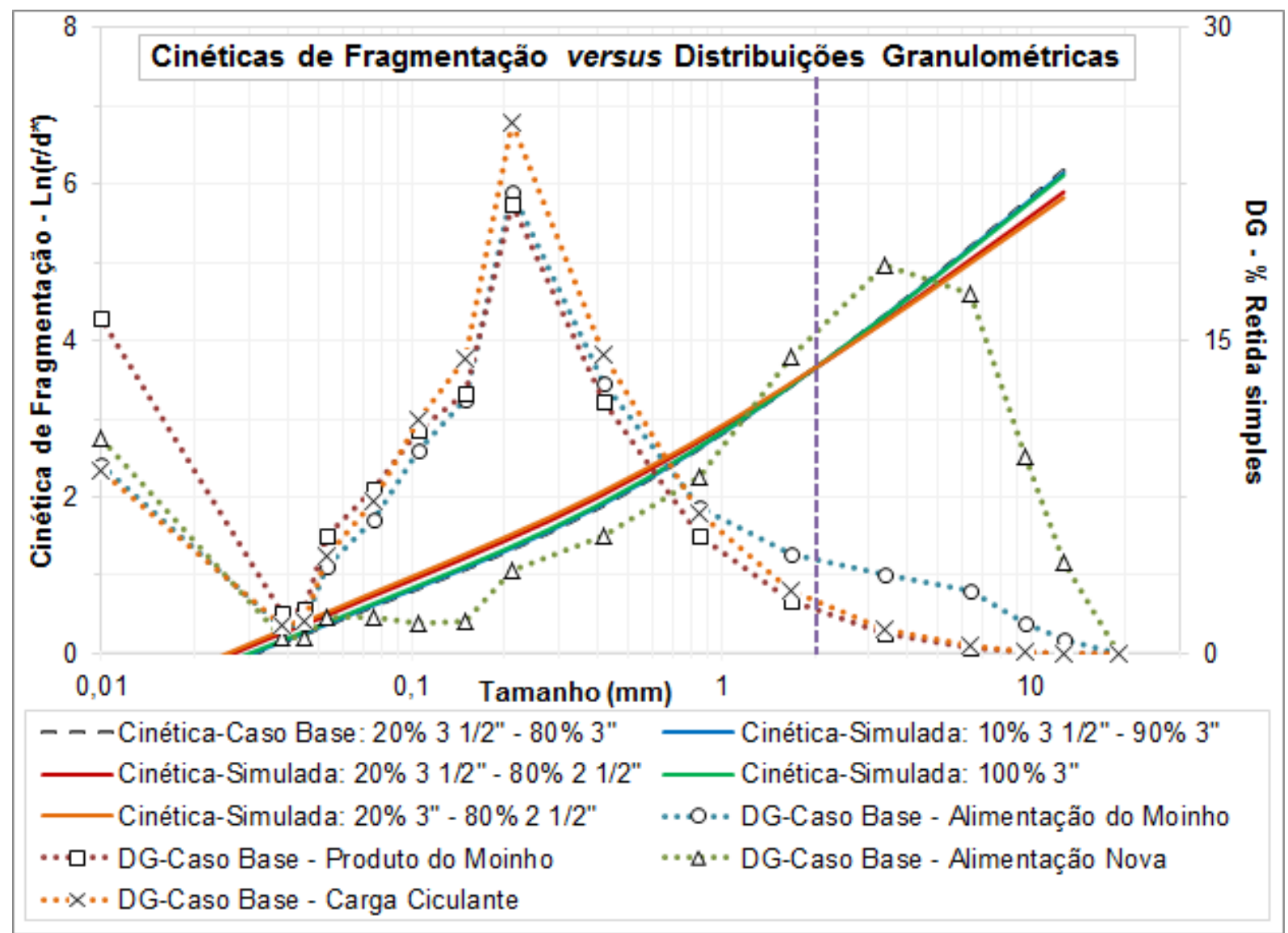

Figura 3. Cinéticas de fragmentação versus distribuições granulométricas.

Nota-se no gráfico da Figura 3 que no tamanho 2,00 mm (linha tracejada lilás) há o cruzamento das curvas de cinéticas de fragmentação simuladas (curvas coloridas) em relação à curva do Caso Base (curva tracejada preta). Assim, para partículas com tamanhos menores que 2,00 mm obtêm-se maior fragmentação para os novos diâmetros de bolas de reposição, principalmente para as configurações com diâmetros de bolas de reposição de 20\% 311/2" - 80\% 21/2" e 20\% 3" - 80\% 21/2". Em contrapartida, ocorre a redução da cinética de fragmentação para as partículas maiores que $2,00 \mathrm{~mm}$ para as respectivas configurações de diâmetros de bolas de reposição simuladas em relação ao Caso Base.

Devido às condições operacionais do circuito de moagem estudado como alta carga circulante, há acúmulo de material das frações intermediárias $-0,420+0,150 \mathrm{~mm}$ no interior do moinho como pode ser observado na curva "DG-Caso Base - Carga Circulante" (curva pontilhada laranja), a qual representa a parcela da carga circulante. Por outro lado, a curva "DG-Caso Base Produto do Moinho" (curva pontilhada vermelha) indica que não há grande acúmulo de 
partículas grosseiras no interior do moinho. Constata-se que para as atuais condições operacionais do circuito de moagem da AGA-Cuiabá há a necessidade de se elevar a fragmentação das frações intermediárias, as quais tendem a apresentar maior tenacidade, de modo a reduzir a alta carga circulante do circuito, possibilitando assim aumento da capacidade produtiva. Contudo, deve-se mitigar a consequente redução de fragmentação das frações grosseiras, provinda da redução dos diâmetros de corpos moedores de reposição do moinho.

\subsection{Ensaios de Moagem em Batelada}

A Tabela 2, a seguir, apresenta as taxas de fragmentação/geração por frações granulométricas calculadas para cada um dos ensaios de moagem realizados. Como os ensaios foram executados nas mesmas condições operacionais com exceção da configuração da carga de bolas é possível comparar as respectivas cinéticas de fragmentação.

Tabela 2. Taxa de Fragmentação/Geração dos ensaios de moagem.

\begin{tabular}{|c|c|c|c|c|c|c|c|c|c|c|}
\hline \multirow{3}{*}{$\begin{array}{c}\text { Fração } \\
\text { Granulométrica } \\
(\mathrm{mm})\end{array}$} & \multicolumn{2}{|c|}{$\begin{array}{c}\text { Caso Base: } \\
20 \% 31 / 2 "-80 \% 3 "\end{array}$} & \multicolumn{2}{|c|}{$\begin{array}{c}\text { Simulado: } \\
10 \% 31 / 2 "-90 \% 3 "\end{array}$} & \multicolumn{2}{|c|}{$\begin{array}{c}\text { Simulado: } \\
20 \% 31 / 2^{\prime \prime}-80 \% 21 / 2^{\prime \prime}\end{array}$} & \multicolumn{2}{|c|}{ Simulado: $100 \% 3^{\prime \prime}$} & \multicolumn{2}{|c|}{$\begin{array}{c}\text { Simulado: } \\
20 \% 3^{\prime \prime}-80 \% 2 \text { 1/2" }\end{array}$} \\
\hline & Ensaio $\mathrm{A}$ & Ensaio B & Ensaio $\mathrm{A}$ & Ensaio $\mathrm{B}$ & Ensaio A & Ensaio B & Ensaio A & Ensaio B & Ensaio $\mathrm{A}$ & Ensaio B \\
\hline & \multicolumn{10}{|c|}{ Taxa de Fragmentação/Geração = (t/h produto) $-($ t/h alimentação) } \\
\hline$+19,1 \mathrm{~mm}$ & 0 & 0 & 0 & 0 & 0 & 0 & 0 & 0 & 0 & 0 \\
\hline$-19,1+12,7 \mathrm{~mm}$ & $-2,3$ & $-3,3$ & $-0,4$ & $-3,1$ & $-0,4$ & $-3,2$ & $-3,5$ & 0,2 & $-2,7$ & $-3,2$ \\
\hline$-12,7+9,50 \mathrm{~mm}$ & $-2,8$ & $-4,5$ & $-1,4$ & $-3,1$ & $-2,1$ & $-4,9$ & $-1,7$ & $-1,5$ & $-4,0$ & $-3,8$ \\
\hline$-9,50+6,35 \mathrm{~mm}$ & $-7,0$ & $-6,5$ & $-6,5$ & $-7,1$ & $-5,8$ & $-5,4$ & $-7,7$ & $-8,2$ & $-1,6$ & $-3,5$ \\
\hline$-6,35+3,35 \mathrm{~mm}$ & $-14,5$ & $-14,6$ & $-14,6$ & $-14,0$ & $-11,1$ & $-12,2$ & $-14,4$ & $-14,5$ & $-11,3$ & $-9,9$ \\
\hline$-3,35+1,68 \mathrm{~mm}$ & $-13,0$ & $-13,0$ & $-13,0$ & $-12,9$ & $-12,5$ & $-12,5$ & $-13,0$ & $-13,0$ & $-12,6$ & $-12,2$ \\
\hline$-1,68+0,850 \mathrm{~mm}$ & $-7,7$ & $-7,6$ & $-7,7$ & $-7,6$ & $-7,8$ & $-7,7$ & $-7,6$ & $-7,7$ & $-7,7$ & $-7,7$ \\
\hline$-0,850+0,420 \mathrm{~mm}$ & $-4,1$ & $-4,1$ & $-4,4$ & $-4,2$ & $-4,8$ & $-4,7$ & $-4,3$ & $-4,4$ & $-4,7$ & $-4,7$ \\
\hline$-0,420+0,210 \mathrm{~mm}$ & 1,6 & 1,6 & 1,0 & 1,6 & $-1,4$ & $-1,2$ & 1,3 & 1,0 & $-1,5$ & $-1,6$ \\
\hline$-0,210+0,150 \mathrm{~mm}$ & 7,0 & 7,4 & 5,8 & 7,6 & 3,9 & 4,5 & 5,7 & 4,4 & 2,2 & 2,0 \\
\hline$-0,150+0,106 \mathrm{~mm}$ & 9,4 & 9,6 & 8,6 & 8,9 & 7,0 & 7,2 & 11,0 & 11,3 & 7,8 & 8,4 \\
\hline$-0,106+0,075 \mathrm{~mm}$ & 7,9 & 8,0 & 7,7 & 7,8 & 7,9 & 8,4 & 6,6 & 6,4 & 6,9 & 7,6 \\
\hline$-0,075+0,053 \mathrm{~mm}$ & 5,8 & 5,4 & 5,5 & 6,2 & 5,8 & 6,6 & 8,9 & 9,0 & 9,5 & 9,5 \\
\hline$-0,053+0,038 \mathrm{~mm}$ & 6,1 & 7,3 & 7,8 & 8,0 & 7,7 & 8,1 & 5,5 & 5,4 & 6,1 & 6,6 \\
\hline$-0,038$ & 13,6 & 14,2 & 11,5 & 11,7 & 13,6 & 16,9 & 13,3 & 11,6 & 13,7 & 12,7 \\
\hline
\end{tabular}

*Valores negativos (azuis) referem-se à fragmentação, enquanto valores positivos (vermelhos) referem-se à geração das partículas nas respectivas frações granulométricas

A Tabela 2 indica que as configurações Caso Base: 20\% 311/2" - 80\% 3", Simulado: 10\% 311/2" - 90\% 3" e Simulado: 100\% 3" apresentaram maiores taxas de fragmentação em relação às configurações Simulado: 20\% 31/2" - 80\% 21/2" e Simulado: 20\% 3" - 80\% 21/2" nas frações granulométricas mais grossas (faixa destacada em laranja). Por outro lado, para a fração granulométrica $-0,420+0,210 \mathrm{~mm}$ (faixa destacada em verde) foram obtidas taxas de fragmentação para as configurações Simulado: $20 \%$ 31/2" - 80\% 21/2" e Simulado: $20 \%$ 3" - 80\% $21 / 2$ ", ou seja, valores negativos indicando desaparecimento de material na respectiva fração granulométrica, enquanto que para a mesma fração granulométrica as configurações Caso Base: 20\% 31/2" - 80\% 3", Simulado: 10\% 31/2" - 90\% 3" e Simulado: 100\% 3" apresentaram valores positivos, indicando acúmulo de material na respectiva fração. 


\section{CONCLUSÃO}

No presente trabalho dois métodos foram utilizados para avaliar a redução dos diâmetros dos corpos moedores no moinho de bolas da AGA-Cuiabá, um por meio de modelagem matemática e simulação e outro mediante ensaios de moagem em batelada em laboratório.

Ambos os métodos utilizados no estudo indicaram que a redução dos diâmetros dos corpos moedores de reposição do moinho industrial da AGA-Cuiabá promovem ganhos significativos em termos da geração de um produto final mais fino e/ou possibilidade de aumento da capacidade.

No entanto, é determinante para este cenário mitigar a consequente redução de fragmentação das frações grosseiras, provinda da redução dos diâmetros de corpos moedores, por meio da redução do top size de alimentação, visto que o circuito de britagem opera com folga.

Os exercícios de simulação e ensaios de moagem em batelada indicaram que a utilização de dois diâmetros de corpos moedores de reposição acarretam em melhor desempenho de moagem do que a utilização de apenas uma diâmetro de corpo moedor. Tal circunstância é notada principalmente em circuitos de moagem em único estágio, onde há necessidade de corpos moedores de grande diâmetro para fragmentar as partículas grossas (quebra de top size) e corpos moedores menores para fragmentar as partículas finas (geração de produto final).

\section{REFERÊNCIAS}

BOND, F. C. Grinding Ball Size Selection. Mining Engineering. May, 1958.

GOMES, W. L. (2014). Integração de Modelos Energéticos e de Balanço Populacional para Simulação de Moagem em Moinhos de Bolas. Dissertação de Mestrado, Escola Politécnica da Universidade de São Paulo.

LYNCH, A. J.; RAO, T. C. (1975). Modelling and Scale-Up of Hydrocyclone Classifiers. Proceedings XI Int Min Proc Congress, Cagliari, 1975.

NAGESWARARAO, K. (1978). Further developments in the modeling an scale up of industrial hydrociclones. PhD Thesis, University of Queensland (JKMRC).

NAGESWARARAO, K. (1995). A generalized model for hydrocyclone classifiers. AusIMM Proceedings, Parkville, December 1995. v. 2, n. 300, 21 p.

WHITEN, W. J. (1976). Ball mill simulation using small calculators. Proceedings AusIMM, p. 47-53. 